\title{
The Role of Using Red-Topped - Non-Additive- Containing - Collecting Tube in Diagnosing Pseudohyperkalemia in Chronic Lymphocytic Leukemia
}

Ahmed Abdulrahim ${ }^{1}$, Mohamedanwar Ghandour ${ }^{2}$, Abu-Bekr Mohamed ${ }^{1}$, Lobelia Samavati $^{3}$

1. Internal Medicine, Wayne State University Detroit Medical Center, Detroit, USA 2. Internal Medicine and Nephrology, Wayne State University Detroit Medical Center, Detroit, USA 3. Pulmonary and Critical Care Medicine, Wayne State University Detroit Medical Center, Detroit, USA

Corresponding author: Ahmed Abdulrahim, ahmedabdulrahim999@gmail.com

\begin{abstract}
Pseudohyperkalemia in the context of chronic lymphocytic leukemia (CLL) is becoming a common clinical presentation in our daily practice, yet the recognition and the overall approach to this condition remains a challenge as clinicians ponder on whether it's a true rise of serum potassium or not, weighing the riskbenefit ratio of giving the full anti-hyperkalemia measures, dreading the potential iatrogenic hypokalemia if it proves to be a pseudohyperkalemia instead.
\end{abstract}

Review began 03/08/2021 Review ended 03/20/2021 Published 03/24/2021

\section{Copyright 2021}

Abdulrahim et al. This is an open access article distributed under the terms of the Creative Commons Attribution License CC-BY 4.0., which permits unrestricted use, distribution, and reproduction in any medium, provided the original author and source are credited.
Categories: Internal Medicine, Nephrology, Oncology

Keywords: hematology-oncology, pulmonary critical care

\section{Introduction}

Pseudohyperkalemia refers to an abnormal elevation in measured serum potassium concentration as a result of transcellular shift during or after the blood specimen has been drawn. Maintaining a high index of suspicion of pseudohyperkalemia is crucial when and where a cautious approach is warranted, especially in the presence of underlying risk factors like leukemia [1], as opposed to hyperkalemia which on the other hand would prompt immediate measures to correct it.

\section{Case Presentation}

The patient is a 54-year-old gentleman, with a history of chronic lymphocytic leukemia (CLL) diagnosed back in 2016, when he received three chemotherapy cycles of bendamustine and rituximab before achieving remission. He lost follow-up afterward and came back to the emergency department (ED) in February 2021 complaining of abdominal dragging sensation towards the left inguinal region and progressive lower limb edema. At the ED, the patient was fully alert and oriented, had massive splenomegaly all the way to the left inguinal region, and pitting bilateral lower limbs edema. His initial work-up showed a white blood cell (WBC) count of $595 \mathrm{~K}$, with an absolute lymphocyte count of $565 \mathrm{~K}$, hemoglobin of $3 \mathrm{gm} / \mathrm{dL}$, and platelets of $56 \mathrm{~K}$. He also had a potassium level of $10 \mathrm{meq} / \mathrm{L}$. The patient had a normal electrocardiogram (EKG) strip upon admission (Figure 1) and normal renal function with urea of $24 \mathrm{mg} / \mathrm{dL}$ and creatinine of $1.2 \mathrm{mg} / \mathrm{dL}, \mathrm{HCO}$ of $22 \mathrm{Mmol} / \mathrm{L}$, and normal anion gap of 6 . He received the full antihyperkalemia measures with calcium gluconate, albuterol aerosol, regular insulin with dextrose $50 \%$, and oral kayexalate with an emergent transfusion of four units of irradiated packed red blood cells (PRBCs) and was transferred to the Medical Intensive Care Unit (MICU) for further management. Of note, the patient was not on any long-term medications, nor was he taking any supplements or herbal medicine. 


\section{Cureus}

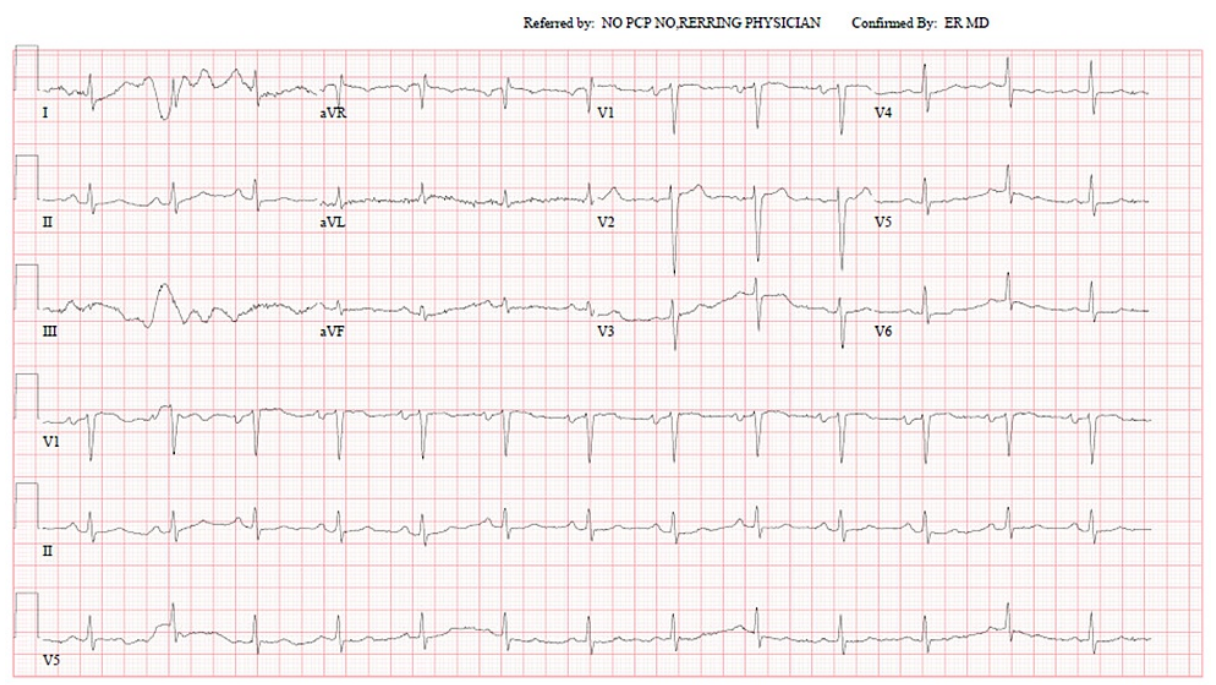

FIGURE 1: Electrocardiogram of the patient upon presentation at the emergency department

Since his admission and over the course of the next six days, the patient remained under the care of MICU service, where he received vigorous IV hydration at 150-200 cc/hour of normal saline, the measured serum potassium level was persistently high, typically at $10 \mathrm{meq} / \mathrm{L}$ for the most part, despite receiving the antihyperkalemia measures for at least three occasions since ED encounter, with a normal EKG strip in the next day and throughout (Figure 2), none of which was demonstrating changes expected in hyperkalemia (Figure 3).

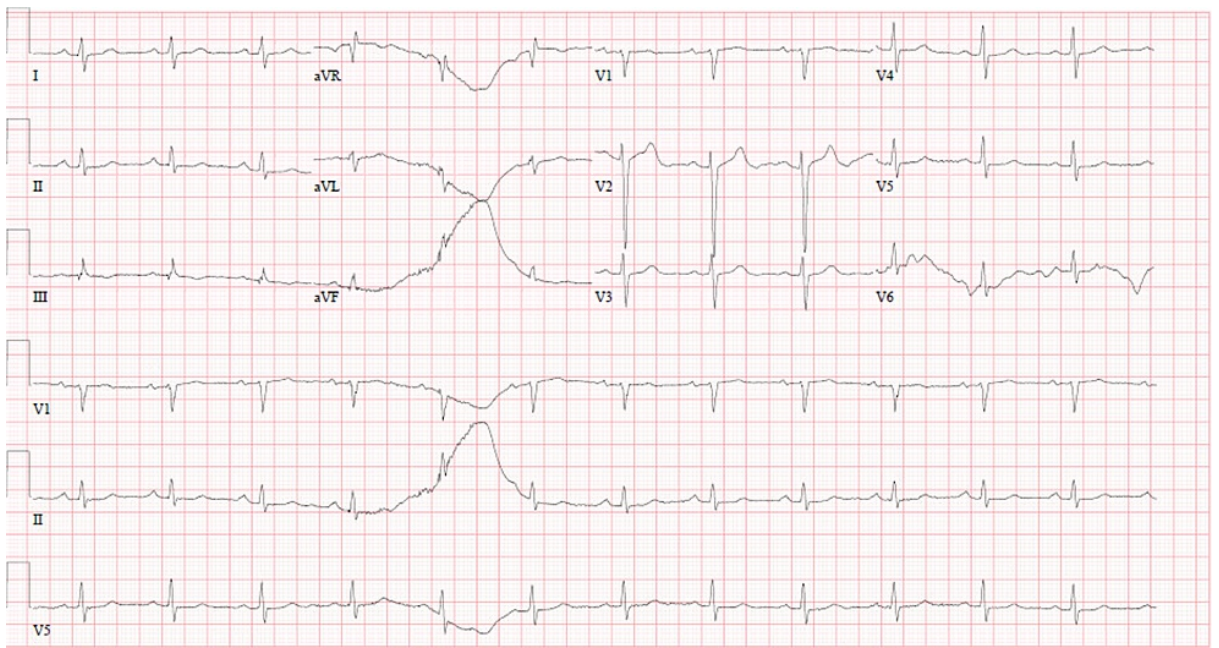

FIGURE 2: Electrocardiogram of the patient on the next day following admission 


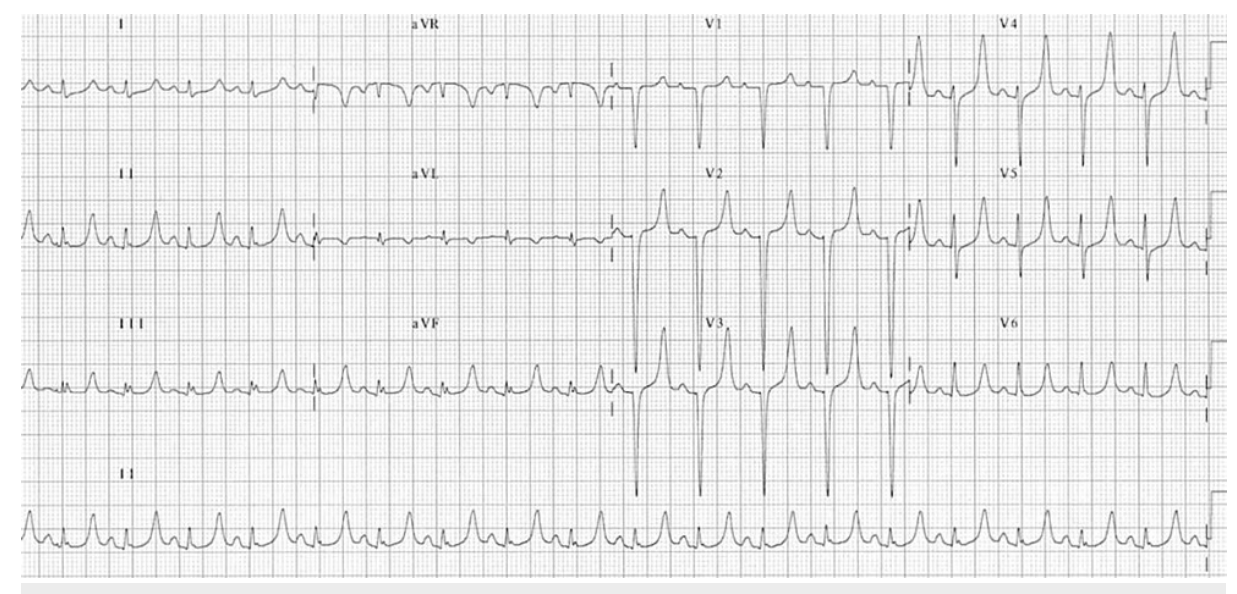

\section{FIGURE 3: Electrocardiogram changes of hyperkalemia demonstrating peak T waves and wide QRS complex in Leads II, III and aVF}

It is noteworthy to mention that magnesium, phosphate, and calcium levels were unremarkable as well. Yet the potassium remained just as high. The oncology team were on board early on and started cytoreductive therapy with prednisolone to reduce tumor burden to a figure reasonable enough before starting chemotherapy and hence reducing the overall risk of tumor lysis syndrome. However, their approach to the persistently high serum potassium level was not different than MICU and ED services; the distinction with pseudohyperkalemia was a challenge given the fragility of the cells and the risk-benefits of giving further hyperkalemia treatment.

On his third day post-admission, an arterial blood gas (ABG) analyzer showed a potassium level of 2.7 meq/L, with PH of 7.43, in stark contrast to the measured serum potassium level of $10 \mathrm{meq} / \mathrm{L}$. At that point onward, the patient did not receive any antihyperkalemia measures and it was deemed to be Pseudohyperkalemia.

On the fifth day post-admission, his serum potassium level came back at $4.7 \mathrm{meq} / \mathrm{L}$ for the first time since his admission. This particular blood sample was sent using the plain red-topped collecting tube that contains no additives. All the serum samples sent using this tube from that point onward showed measured serum potassium levels between 3.9-4.7 meq/L. All the blood samples before, with high measured serum potassium levels, were sent using the standard green-topped collecting tube with lithium heparin additives. The patient's level of care was de-escalated to the medical floor.

On this eleventh day post-admission, the measured serum potassium level went up to 10 from $3.9 \mathrm{Meq} / \mathrm{L}$, only 10 hours apart, and it was because of the use of a green-topped tube. A repeat blood sample was sent using a red-topped tube and the measured serum potassium level came back at $4.1 \mathrm{meq} / \mathrm{L}$.

\section{Discussion}

Pseudohyperkalemia in the context of significant leukocytosis is presumed to be secondary to increased cell lysis with the liberation of intracellular potassium onto the bloodstream, especially when cell fragility is suspected in increased blood cellular component, like in leukemias [1]. However, the etiology of cellular lysis had been stipulated to a multitude of factors that pertain more to the maneuvers upon acquiring the blood samples. The use of a pneumatic tube transport system for example is implicated in producing pseudohyperkalemia [2], the exposure of the blood samples to variant ambient temperature [3], and delayed processing which exhausts the available glucose to generate adenosine triphosphate (ATP), which fuels the $\mathrm{Na} / \mathrm{K}$ pump maintaining the gradient across the cell membrane. ATP shortage and consequent pump failure will result in leakage of potassium out of the cell, resulting in pseudohyperkalemia [4].

Ascertaining the diagnosis of pseudohyperkalemia is of utmost importance given the implications of undertaking aggressive anti-hyperkalemia measures in such context and inflicting dangerous iatrogenic hypokalemia when a cautious expectant management is far more appropriate. Ideally, this shall begin at the ED portal, when and where a high index of suspicion shall be maintained in the absence of risk factors. Notably, apart from exogenous (injectable) hyperkalemia, risk factors for hyperkalemia almost always exist; for example in renal impairment, rhabdomyolysis, metabolic acidosis, and tumor lysis syndrome. Moreover, EKG changes are expected to occur with the incremental increase in serum potassium level, the higher the serum potassium level, the higher the chances of detecting new EKG changes [5]. The absence of hyperkalemia risk factors, EKG changes, and congruent metabolic disturbances with hyperkalemia should prompt astute clinicians to consider pseudohyperkalemia instead, especially in conditions where cells fragility and hyperkalemia are more prevalent [1] - like in leukemias - than otherwise. 
Many tools had been suggested to diagnose pseudohyperkalemia when approaching CLL: measuring potassium on arterial blood gas [6], correlating serum potassium level with the plasma potassium level, as more often than not, the former tends to be higher [7], and avoiding the use of pneumatic tube delivery system to reduce the sheer and mechanical force on the already fragile CLL cells [8].

As it had shown in the patient in reference, amid an exceedingly high measured serum potassium of 10 , which persisted following repeated administration of the anti-hyperkalemia protocol therapy, pseudohyperkalemia was suspected because of the inconsistency of the overall picture - normal phosphate, EKG, HCO3, PH, and unrelenting serum potassium level following repeated administration of antihyperkalemia treatment protocol. The suspicion grew further following the obtainment of the ABG and having a poorly correlated potassium level to that of the serum. In fact, using the ABG analyzer to assess the potassium level when the serum potassium level is high affords a quick and reliable mean to corroborate serum potassium level, as it has been estimated that a mean difference of $0.14 \mathrm{mmol} / \mathrm{L}$ exists between serum and ABG analyzer potassium level [9], yet the difference, in this case, was much higher. This confusion was sorted out when the blood sample was sent using the red-topped collecting tube.

This report argues the invaluable use of the red-topped, $6 \mathrm{ml}$ Vacuette ${ }^{\circledR}$ (Greiner Bio-One International $\mathrm{GmbH}$, Kremsmünster, Austria) - non-additive-containing - collecting tube in CLL instead of the standard green-topped, $5 \mathrm{ml}$ Vacuette ${ }^{\circledR}$ - lithium heparin containing - collecting tube that is used for chemistry panels, whenever the distinction between pseudohyperkalemia and hyperkalemia is challenging (Figure 4). Before resorting to this, we worked with nursing staff and educated them on the necessity of careful withdrawal of blood and expedient delivery of the sample to the laboratory and having it run on stat basis such rigorous efforts have proven to be time/labor-consuming and unyielding. The ABG analyzer was a quicker approach initially, but the red-topped Vacuette ${ }^{\circledR}$ collecting tube gave us a closer look into the sought-after serum potassium level amid an active relapsed chronic lymphocytic leukemia.

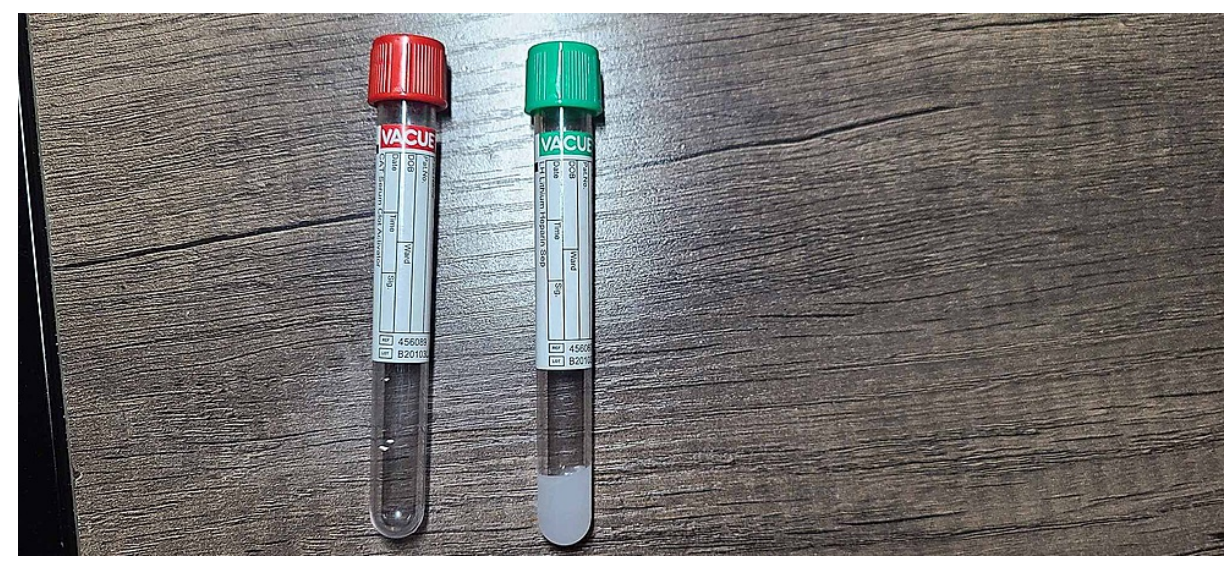
FIGURE 4: Red-topped - without additive - collecting tube (left) and the
green-topped - lithium heparin containing - collecting tube (right)

\section{Conclusions}

Pseudohyperkalemia is a common finding in myeloproliferative disorder that may lead to inappropriate management of patients, yet not well recognized by medical health providers as it should, much less having the appropriate understanding to use available resources to tackle it. This case report emphasizes the importance of utilizing the particular red-topped - non-additive-containing - collecting tube as a safe, reliable, and timely approach when diagnosing pseudohyperkalemia in patients with chronic lymphoblastic leukemia.

\section{Additional Information}

\section{Disclosures}

Human subjects: Consent was obtained or waived by all participants in this study. Conflicts of interest: In compliance with the ICMJE uniform disclosure form, all authors declare the following: Payment/services info: All authors have declared that no financial support was received from any organization for the submitted work. Financial relationships: All authors have declared that they have no financial relationships at present or within the previous three years with any organizations that might have an interest in the submitted work. Other relationships: All authors have declared that there are no other relationships or activities that could appear to have influenced the submitted work.

\section{References}




\section{Cureus}

1. Sevastos N, Theodossiades G, Savvas SP, Tsilidis K, Efstathiou S, Archimandritis AJ: Pseudohyperkalemia in patients with increased cellular components of blood. Am J Med Sci. 2006, 331:17-21. 10.1097/00000441200601000-00006

2. Kotla S, Makhoul S, Gonzalez L: Pseudohyperkalemia from a pneumatic tube transport system: case report and literature review. Hosp Pharm. 2012, 47:371-4. 10.1310/hpj4705-371

3. Sinclair D, Briston P, Young R, Pepin N: Seasonal pseudohyperkalaemia. J Clin Pathol. 2003, 56:385-8. 10.1136/jcp.56.5.385

4. Asirvatham JR, Moses V, Bjornson L: Errors in potassium measurement: a laboratory perspective for the clinician. N Am J Med Sci. 2013, 5:255-9. 10.4103/1947-2714.110426

5. Montague BT, Ouellette JR, Buller GK: Retrospective review of the frequency of ECG changes in hyperkalemia. Clin J Am Soc Nephrol. 2008, 3:324-30. 10.2215/CJN.04611007

6. Ruddy KJ, Wu D, Brown JR: Pseudohyperkalemia in chronic lymphocytic leukemia . J Clin Oncol. 2008, 26:2781-2. 10.1200/JCO.2008.16.3014

7. Handy BC, Shen Y: Evaluation of potassium values in a cancer patient population . Lab Med. 2005, 36:95-7. 10.1309/18JAGL5KAYQWP8BL

8. Huang N, Bufalino S, Czerlanis C: Pneumatic tube-induced reverse pseudohyperkalemia in a patient with chronic lymphocytic leukemia. Fed Pract. 2016, 33:60-2.

9. Gupta S, Gupta AK, Singh K, Verma M: Are sodium and potassium results on arterial blood gas analyzer equivalent to those on electrolyte analyzer?. Indian J Crit Care Med. 2016, 20:233-7. 10.4103/09725229.180044 